\title{
Constraints for dark matter local substructure properties from dynamic System-DM substructure interactions
}

\author{
ALMA XOCHITL GONZALEZ MORALES* \\ Instituto de Ciencias Nucleares, UNAM, A.P. 70-543, 04510, Circuito Exterior C.U., D.F., \\ Mexico. \\ E-mail: alma.gonzalez@nucleares.unam.mx

\section{OCTAVIO VALENZUELA} \\ Instituto de Astronomia, UNAM, A.P. 70-264, 04510, Circuito Exterior C.U., D.F., Mexico. \\ E-mail: octavio@astro.unam.mx
}

\section{LUIS AGUILAR}

Instituto de Astronomia, UNAM, A.P. 877, 22860. Ensenada, B.C., Mexico

E-mail: aguilar@astrosen.unam.mx

\begin{abstract}
We explore the possibility to constrain the dark matter (DM) substructure properties, mass and distribution, at subgalactic scales, by considering the dynamics of some astrophysical systems under the dynamical influence of such substructure. We present preliminary results discussing the sensitivity of the Solar System dynamics to the small scale DM distribution.
\end{abstract}

VIII International Workshop on the Dark Side of the Universe

June 10-15, 2012

B?zios, Rio de Janeiro, Brasil

\footnotetext{
*Speaker.
} 


\section{Introduction}

The $\Lambda$-CDM cosmological paradigm is consistent with the large scale observations of the universe; however, one of the main ingredients, the Dark Matter (DM), has not yet been detected. On the other hand, DM models based on WIMP's predict a large abundance of substructure at subgalactic scales. This has been considered as a potential problem for the $\Lambda$-CDM scenario because it is not obvious that the predicted dark substructure has an observational counterpart [ $[2]$, see however [B]].

The properties, mass and abundance, of the substructure could be related to the properties of the power spectrum for primordial fluctuations, the nature of the dark matter, and the dynamical evolution of the fluctuations. In the $\Lambda$-CDM model the primordial mass power spectrum is fixed by inflation, and the dynamical evolution is followed trough $N$-body simulations, so that to constrain the properties of the substructure can constitute a test for the nature of the DM particle. Once a specific DM particle is assumed, the survival of the smallest structures trough the structure formation history is by itself a topic of discussion, being the extrapolation from $\mathrm{N}$-body simulations at larger scales and the semi-analytic models the most used tools used to estimate the amount of substructure that could be present, in the galactic halos today [ [ [, [].

Also, it has been shown that the free streaming, and/or the kinetic decoupling, of WIMP's like DM candidates leads to a mass-power spectrum cut off at approximately $M_{\min } \sim 10^{-4}-10^{-12} M_{\odot}$ [目, 团], while in the case of the Axion the cut off could be as small as $10^{-18}-10^{-20} M_{\odot}\left[\mathbb{Q}\right.$, 团]..$^{1}$ This small scale cut-off is unreachable to study with current $N$-body simulations, or with analytical models, and it is also undetectable through current observational techniques.

Here we explore an strategy that could allow us to set upper limits to the existence of the dark small structure - micro-halos and streams - in the galactic halo. Since the orbital elements of planets in our Solar System are known with outstanding accuracy [W], they might be sensitive to dynamical perturbations triggered by dark matter substructure. In this work we focus on the variation of the astronomical unit, due to the presence of dark substructure and how this compares with the reported anomaly in [15]]. We also study the evolution of the Earth-Moon system, under the same perturbation field, and compare our results with the current uncertainty with which the

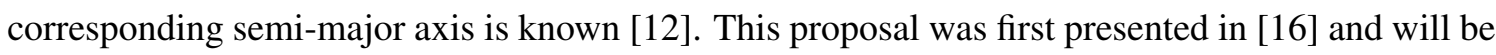
presented with more detail in [ㅁ] ].

\section{Target tidal interactions with mini-halos and streams}

The dynamics of some astrophysical systems is known with great accuracy that allows to constrain the existence of incredibly small gravitational perturbations. Here we consider the dynamical perturbations that such systems (the target) could undergo in the presence of dark substructure. That could be in the form of mini-halos or streams, - former DM sub-halos that had been already disrupted by tidal forces or encounters- - We will treat the encounters with mini-halos, and streams, as independent of each other and separately. First, we present the equations we will use for inde-

\footnotetext{
${ }^{1}$ Other DM candidates like the Warm Dark Matter are also consistent with the large scale but they predict a PS cut off at approximately the dwarf galaxies mass [ए]]).
} 
pendent single encounters, and we then explain how we follow the evolution of the target under multiple encounters.

\subsection{One single encounter with the target}

Our first approach is to consider spherical micro-halos: the impulsive energy injection to a target orbit during one single encounter, is

$$
\frac{\langle\Delta E\rangle_{m h}}{\left|E_{b}\right|}=\frac{14 G M_{p}^{2}}{3 M_{c} v_{0}^{2} p^{4}} a^{3},
$$

where, the impact parameter $p$ is the minimal distance between the target and the pertuber; $M_{p}$ is the perturber mass; $M_{c}$ is the mass of the central body in the target; $v_{0}$ is the relative encounter velocity; and $a$ is the mean separation of the target system (it coincide with the semi-major axis for some systems) [18, [प]. Equation [ 2 is valid whenever the characteristic size of the perturber is smaller that the impact parameter (point mass approximation); when the impact parameter is much larger than the extent of target (distant tide approximation); and the encounter velocity is much larger than the internal velocity of the target (impulsive regime). We have verified that the systems under study falls in these approximations.

In our second approach, we consider the perturbers to be a sort of dark streams of cylindrical symmetry, characterized by their linear mass density. The simplest model for a stream is 1-dimensional, i.e. a line of linear mass density $\lambda_{S T}$. In this case, the energy injection normalized to the binding energy of the target is:

$$
\frac{\langle\Delta E\rangle_{S T}}{\left|E_{b}\right|}=\frac{4 \pi^{2} G \lambda_{S T}^{2}}{M_{c} v_{0}^{2} p^{2} \sin ^{2}(\theta)} a^{3}\left(\cos ^{2}(\alpha) \cos ^{2}(\psi)+\sin ^{2}(\psi)\right) .
$$

The angles $\alpha$ and $\psi$ defines the orientation of the target orbital plane, and $\theta$ the relative direction of movement of target, both with respect to the stream. More realistic models include the finite cross section stream with constant density; or with a power law density, either with a cusp or a core in the center. These models reduces to the one dimensional when the impact parameter is larger than the cross-section of the stream. Here we will consider only the 1-dimensional case and the analysis for the other models, as well as the derivation of equation [2.2], will be presented in [ए]].

\subsection{Multiple encounters during the lifetime of the target}

As a result of multiple encounters, either with mini-halos or streams, the fractional energy of the target system will change. Our general approach to study the effect of this multiple encounters consists of Monte Carlo experiments. First, we draw random values for the encounter parameters ( $p, v_{0}, \alpha$, etc.), and use them to assess the effect of one single encounter trough the fractional energy, $\langle\Delta E\rangle / E_{b}$. We then increment the experiment timer with $\Delta t_{e n c} \propto 2 p / v_{0}$ (the time it takes the perturber to traverse a sphere of radius equal to the impact parameter at the encounter velocity), and then we continue sampling encounters until the time limit is reached, (the lifetime of the target), or until the fractional energy reaches the unity (i.e. the target gets unbound). We repeat this procedure a number of times, so that we can construct an ensemble of histories of the multiple encounters 
between the target and the perturber population. Finally, we find the median value of the total dynamical perturbations to the target experiment due to the presence of the dark substructure.

In the case of encounters with mini-halos we sample the impact parameters from a nearestneighbor distribution, with numerical density $v=\rho_{d m} f_{s} / m_{m h},\left(f_{s}\right.$ is the fraction of the mass density, $\rho_{d m}$, of dark matter that is in form of mini-halos of mass $m_{m h}$ ). On the other hand, if the perturbers are streams, we use the cumulative distribution shown in figure $\mathbb{W}$. The problem in using a nearest neighbor distribution, again, is that we would be linking a sample volume to the impact parameter distribution. To break this link, we start with a given random realization of a region of the 3D-space where lines have been thrown at random and uniformly. Then, we choose a collection of arbitrary, and sufficiently, separated points within this region, and determine the minimum distance, impact parameter, for each point to the lines, ending with the distribution in figure $\mathbb{W}$. Finally, we relate the starting realization to a specific universe with a certain spatial mass density, and linear mass density for the lines, with the relation, $p=\left(\sqrt{\lambda_{S T} \rho^{*} / f_{S} \rho_{d m}}\right) x$, where $\rho^{*}=1205.08$ is the numerical mass density we measure from the random sampling, and the other parameters depend on the specific system under study. This relation comes from a dimensional analysis. For the velocity distribution, we use a Maxwellian distribution, and a uniform distribution function for the geometric parameters, ( $\beta, \alpha$, etc.). In the next section we present a couple of examples that illustrates the applicability of the method we have described.

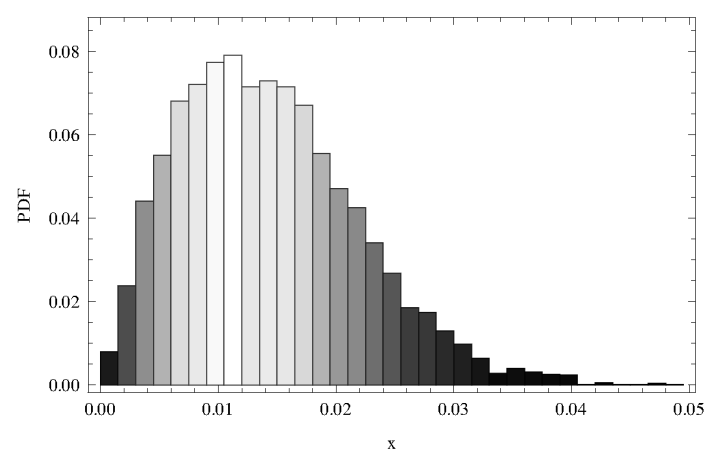

Figure 1: Probability distribution function of impact parameters for stream like perturbers. The impact parameter is scaled according to the particular physical system using: $p=\left(\sqrt{\lambda_{S T} \rho^{*} / f_{S} \rho}\right) x$

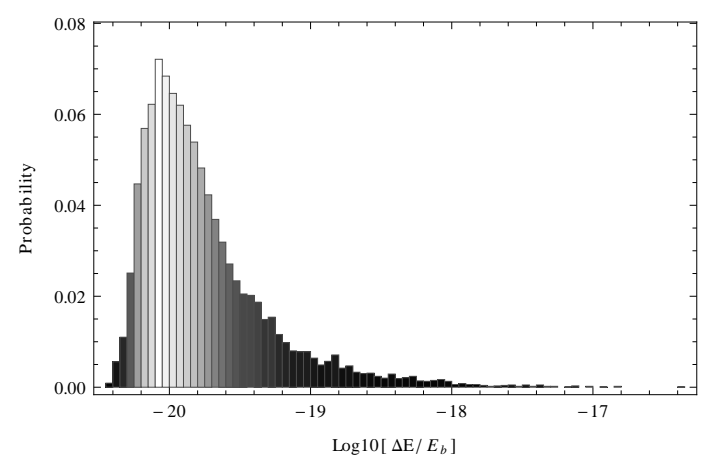

Figure 2: Probability distribution function for the Earth-Moon system binding energy change. Each run in the ensemble correspond to the result after multiple encounters with mini-halos of mass $m_{m h}=$ $10^{-6} M_{\odot}$ for about $4 G y r$, and with the canonical value of the dark matter local density.

\section{Examples of applicability}

The Solar System dynamics is known with high accuracy. The planetary semi-major axis is known with a precision of the order of $\Delta a / a \approx 10^{-8}$ for Neptune [ $[$ ]], while for the Earth-Moon system is $\approx 10^{-12}$ [ए2]]. This precision has made the Solar System to be a great laboratory for tests of general relativity and, in this work, for constraint the amount of DM substructure that may be present in the solar neighborhood. 


\subsection{The case of the Earth-Moon system}

In figure, $\square$ we show an example of the resultant probability distribution for an ensemble of Monte-Carlo experiments. Each of the runs in the ensemble corresponds to a history of encounters between the Earth-Moon system and mini-halos, (with $m_{S T}=10^{-6} M_{\odot}$ ), for about 4 Gyr. Since the distribution is non-symmetric, we will use the median value instead of the mean. A similar distribution is obtained when the perturbers are streams.

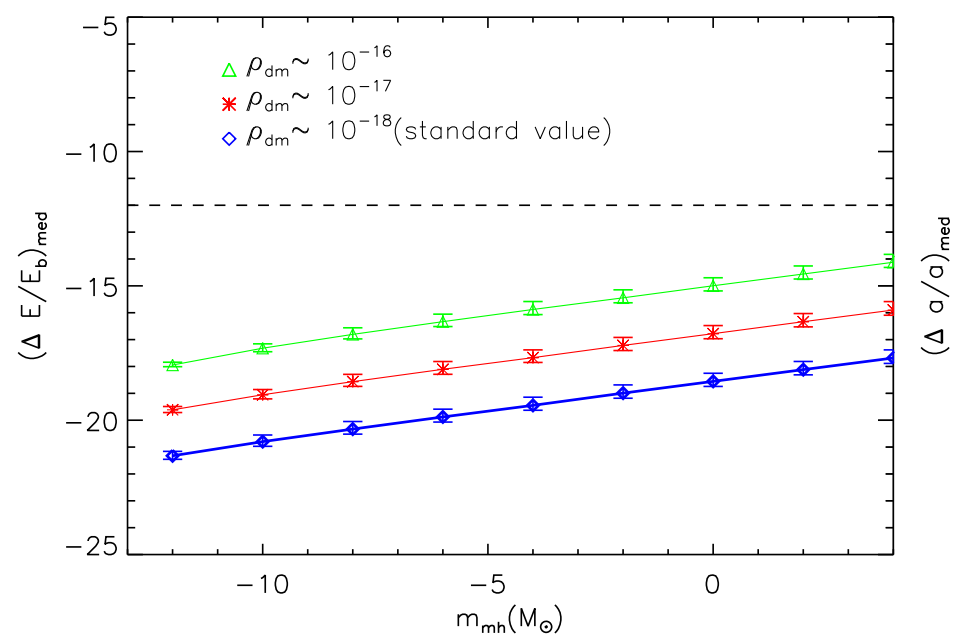

Figure 3: Median value of the Earth-Moon system binding energy total change as a result of multiple encounters with mini-halos of mass $m_{m h}$, and for three different values of the local dark matter density (in $M_{\odot} / A U^{3}$ units). The dashed line correspond to the current observational limit [ए]]

Now, in figure $[$ we show the results for the median value of the energy perturbation, as a function of the mini-halo mass, (spherical), and for three different values of the local dark matter density, including the canonical value (blue line), $\rho_{d m} \sim 10^{-18} M_{\odot} / A U^{3}\left(0.3 \mathrm{GeV} / \mathrm{cm}^{3}\right.$ ) [[3], [4]]. We can see that the system is not very sensitive to this kind of perturbations, not even at higher values of the local dark matter density. On the other hand, in figure $⿴ 囗 ⿱ 一 一)$ we show the results now as a function of the mass linear density, for stream like perturbers. Note that values $\lambda_{S T}>10^{-10} M_{\odot} / A U$ are excluded by the current precision in the Earth-Moon distance (dashed line), if the fraction of streams is assumed to be the unity. Lowering this last quantity to the $10 \%$, i.e for $f_{s}=0.1$, lowers the factor $\Delta E / E_{b}$ to an approximately $15 \%$ of its value for $f_{s}=1$. Even with such a small fraction of the dark matter in form of streams, the allowed values for the linear density are for $\lambda_{S T}<10^{-8}$. Only for $f_{s} \approx 0.05$ the values $\lambda_{S T}<1 M_{\odot} / A U$ are allowed by the observational restrictions. Combinations with larger values of the the local dark matter density are also excluded, for $f_{s}=1$.

\subsection{Anomalous evolution of the astronomical unit}

There is some evidence of a variation in the astronomical unit (AU), the increase is reported to be about $15 \mathrm{~cm} \mathrm{yr}^{-1}$ [다]. This anomaly remains unexplained, (see however [ㅁ]).

We have applied our formalism of multiple encounters with dark substructure to the Sun-Earth system, to check if such anomaly could be explained by the dynamic perturbations due to the 


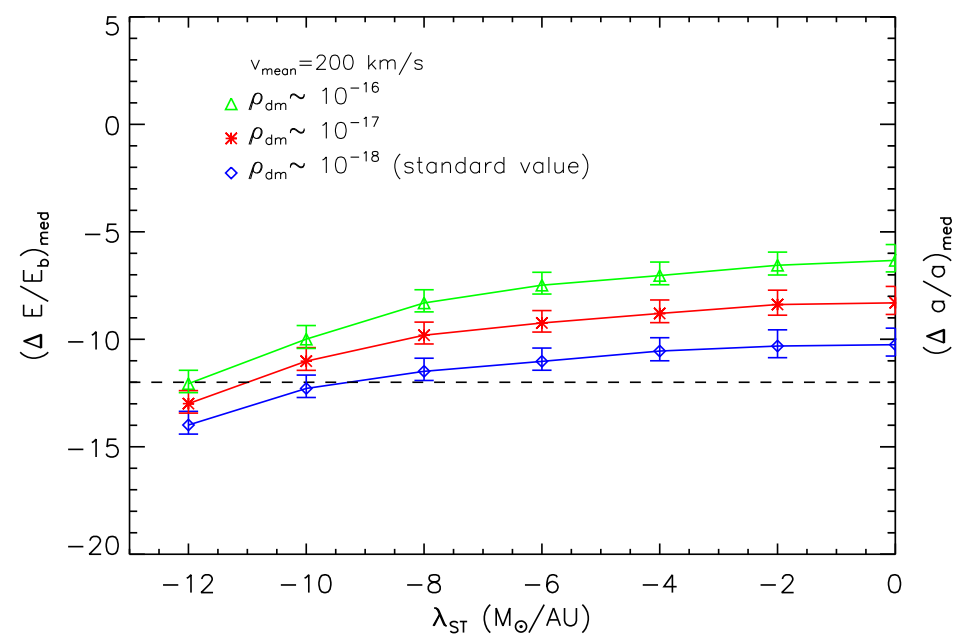

Figure 4: Median value of the Earth-Moon system binding energy total change as a result of multiple encounters with streams mass linear density $\lambda_{S T}$, and for three different values of the local dark matter density (in $M_{\odot} / A U^{3}$ units). The dashed line correspond to the current observational limit [ए2]

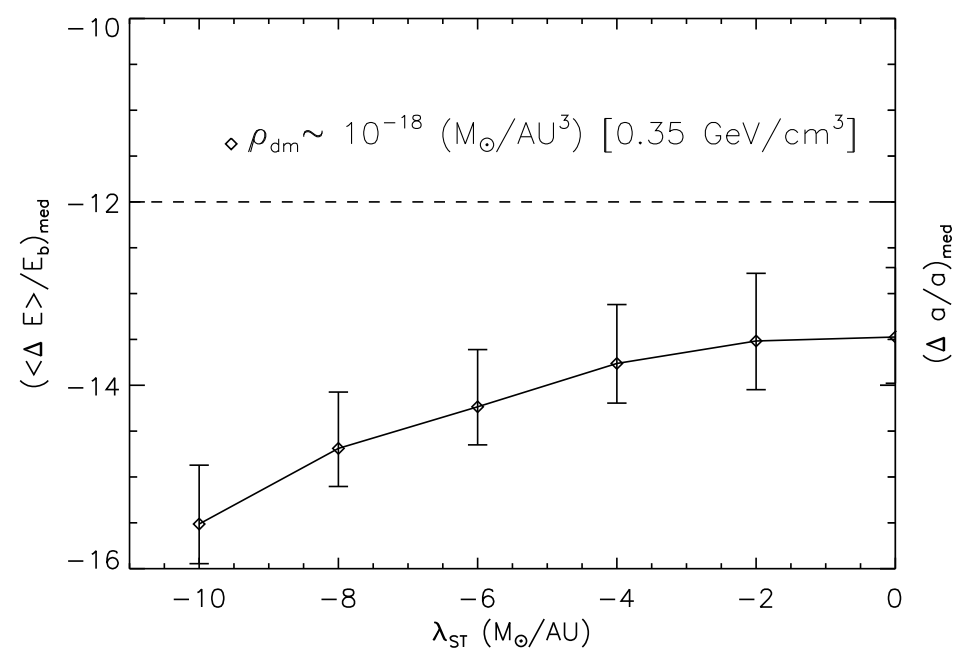

Figure 5: Median value of the Earth-Sun system binding energy change, as a result of multiple encounters with streams with linear mass density $\lambda_{S T}$. The dashed line correspond to the reported variation of the AU $(\approx 15 \mathrm{~cm} / \mathrm{yr})$.

presence of dark substructure. In figure $\square$ we show the median value of the total fractional energy for different values of the perturber linear density. We see that in general these kind of perturbations are not enough to explain the reported anomaly, indicated by the dashed line. A similar trend was found when studying interactions with mini-halos. 


\section{Discussion}

We studied the dynamical effect that a population of dark substructure, mini-halos or streams, could imprint in the dynamics of astrophysical systems. In particular, the dynamics of the Solar System. And how this could be used to set up constraints to the properties, mass and distribution, of such substructure.

We find, for the Earth-Moon system, the perturbations due to the presence of mini-halos are smaller than the observational restrictions, so that we can not impose any constraint to their mass. Even in the idealized case that all the dark matter density is distributed in mini-halos, the cumulative effect is smaller that the current restrictions. On the other hand, we find several restrictions to the value of the linear mass density of streams that could be present in the solar neighborhood with out being in contradiction with the current observational limits. This values depends on the fraction of the dark matter being in the form of streams. For $f_{s}=1$ only values of $\lambda_{S T}<10^{-10} M_{\odot} / A U$ are allowed, while for $f_{s} \approx 0.05$ larger values are permitted, $\lambda_{S T}<1 M_{\odot} / A U$. We also find that neither the perturbations due to mini-halos nor due to streams, for about the lifetime of the Earth, would be energetic enough to explain the reported anomaly in its variation.

But, we would like to remark that those results are sensitive to the mean value of the local dark matter density, which is determined at larger scales (about Kilo parsecs). However, it remains open the possibility of having large over-densities at lower scales, still consistent with the mean density. Increasing the value of the dark matter density has the effect of increasing the total fractional energy given, so it would be possible to explain the anomaly in that case.

In this contribution we have considered that all the dark matter is in form of mini-halos, or streams, separately. A more realistic situation would be one in which a fraction of the mass is in form of mini-halos, and the rest is in form of streams. Such cases, and their implications, as well as the study of other systems, are considered in a work that is under revision now. We will also discuss a possible connection between the value of the linear mass density and the mass of the initial sub-halos. That could allow us to translate our constraints, to information about the cut-off mass power spectrum.

\subsection{Acknowledgments}

Alma wants to thanks the DSU organizers, and the ICN-UNAM teaching department, for all the financial support when attending at this conference. Alma's work is supported by DGAPAUNAM grant No. IN115311 and a CONACyT PhD fellowship.

\section{References}

[1] Green, A. M. 2010, arXiv:1004.2383

[2] Klypin, A., Kravtsov, A. V., Valenzuela, O., and Prada, F. 1999, ApJ, 522, 82

[3] Xu, D. D., Mao, S., Cooper, A. P., et al. 2010, MNRAS, 408, 1721

[4] Diemand,J. and others, Clumps and streams in the local dark matter distribution, Nature 454 (2008) 735-738.

[5] R. E. Angulo and S. D. M. White, The Birth and Growth of Neutralino Haloes, arXiv: 0906.1730. 
[6] Green,A. M., Hofmann,S. and Schwarz,D. J. The power spectrum of SUSY - CDM on sub-galactic scales, Mon.Not.Roy.Astron.Soc. 353 (2004)astro-ph/0309621.

[7] Profumo, S., K. Sigurdson, and M. Kamionkowski, What mass are the smallest protohalos?, Phys.Rev.Lett. 97 (2006) 031301,astro-ph/0603373.

[8] M. C. Johnson and M. Kamionkowski, Dynamical and Gravitational Instability of Oscillating-Field Dark Energy and Dark Matter, Phys.Rev. D78 (2008) 063010, arXiv : 0805 . 1748.

[9] Barranco, J. and Bernal,A., Self-gravitating system made of axions, Phys.Rev. D83 (2011) 043525,arXiv: 1001.1769.

[10] P. Colin, V. Avila-Reese, and O. Valenzuela, Substructure and halo density profiles in a warm dark matter cosmology, Astrophys.J. 542 (2000) 622-630,astro-ph/0004115

[11] E. Pitjeva and N. Pitjev, Estimations of changes of the Sun's mass and the gravitation constant from the modern observations of planets and spacecraft, arXiv: 1108.0246.

[12] T. W. Murphy, and others, APOLLO: millimeter lunar laser ranging, Classical and Quantum Gravity 29 (2012) 184005.

[13] P. Salucci, F. Nesti, G. Gentile, and C. Martins, The dark matter density at the Sun's location, Astron.Astrophys. 523 (2010) A83, [arX1V:1003.3101].

[14] W. de Boer and M. Weber, The Dark Matter Density in the Solar Neighborhood reconsidered, JCAP 1104 (2011) 002, [arXiv:1011,6323].

[15] J. D. Anderson and M. M. Nieto, Astrometric solar-system anomalies, in IAU Symposium (S. A. Klioner, P. K. Seidelmann, and M. H. Soffel, eds.), vol. 261, p. 189, 2010.

[16] González-Morales, A. X., Valenzuela, O., \& Aguilar, L. 2011, Revista Mexicana de Astronomia y Astrofisica Conference Series, 40, 15

[17] Gonzalez-Morales Alma X., Valenzuela, O. and Aguilar, L. A., The Dynamics of astrophysical systems as a constraint to DM Substructure, in preparation

[18] Binney, J. and Tremaine,S. , Galactic Dynamics: Second Edition. Princeton University Press, Princeton, NJ USA, 2008., 2008.

[19] L. A. Aguilar and S. D. M. White, Tidal interactions between spherical galaxies, ApJ 295 (Aug., 1985) 374-387.

[20] L. Iorio, An Empirical Explanation of the Anomalous Increases in theAstronomical Unit and the Lunar Eccentricity, Astron.J. 142 (2011) 68, [arXiv: 1102 .4572].

[21] X. Hernandez and W. H. Lee, The tightening of wide binaries in dSph galaxies through dynamical friction as a test of the Dark Matter hypothesis, arXiv:0803.1507.

[22] Penarrubia, J. et. al., Binary stars as probes of dark substructures in dwarf galaxies, arXiv: 1005.5388 .

[23] M. G. Walker, M. Mateo, E. W. Olszewski, O. Y. Gnedin, X. Wang, et. al., Velocity Dispersion Profiles of Seven Dwarf Spheroidal Galaxies, arXiv:0708.0010. 